\title{
Galaxy formation and cosmology in the ELT era
}

\author{
Patrick J. McCarthy \\ Carnegie Observatories 813 Santa Barbara St. Pasadena, CA 91101, USA \\ pmc2@ociw.edu
}

\begin{abstract}
Understanding the energy and matter content of the Universe and its evolution are key goals for the international astronomical community and all extremely large telescope projects. ELTs will bring a wide range of tools to bear on questions relating to the nature of dark energy and the formation and early evolution of galaxies. Natural seeing and ground-layer corrected instruments on ELTs will quantify the assembly of galaxies at modest redshifts, while near-IR diffraction-limited instruments will probe the internal structure of early galaxies and the epoch of first light and reionization.
\end{abstract}

Keywords. Cosmology: early universe, Galaxies: formation, evolution

\section{Introduction}

The scientific backdrop for extremely large telescope (ELT) studies in the US is provided by the National Academy of Science's decadal survey and the US Department of Energy's "Quarks to the Cosmos" reports. These two reports layout the top science priorities in astrophysics and several related directly to cosmology and galaxy evolution studies with large aperture telescopes. Of particular relevance are the goals of understanding the nature and distribution of dark energy and dark matter, determining the expansion history of the Universe from the Big Bang to the present and, identifying the first stars and stellar systems. Survey activities throughout the broader international astronomical community have identified similar priorities. These activities have led to a number of documented scientific justifications for ELTs and cosmology and galaxy evolution studies figure prominently in all. The present discussion is in large part distilled from the European ELT science case, the GSMT science case and the deliberations of the science working group for the Giant Magellan Telescope project in the US.

\section{Cosmology with ELTs}

A key challenge in observational cosmology today is understanding the source of the accelerating expansion and, hence, the nature of dark energy. Astronomical probes of dark energy are focused on determination of the equation of state and its time evolution. This involves measuring the expansion history from luminosity or angular-size distances to one or more class of standard, or calibrated, source or rod. At visible and near-IR wavelengths Type 1a SNe offer the currently viable path to determinations of the expansion history. Baryon acoustic oscillations offer an interesting approach to determinations of the angular size distance and an independent approach to a direct determination of the evolution of the equation of state. Baryon oscillation experiments at $1<z<3$ (e.g. Blake \& Glazebrook 2003) require survey areas and depths that are within reach of current facilities. These studies are probably more appropriate for $8 \mathrm{~m}$ class wide-field 


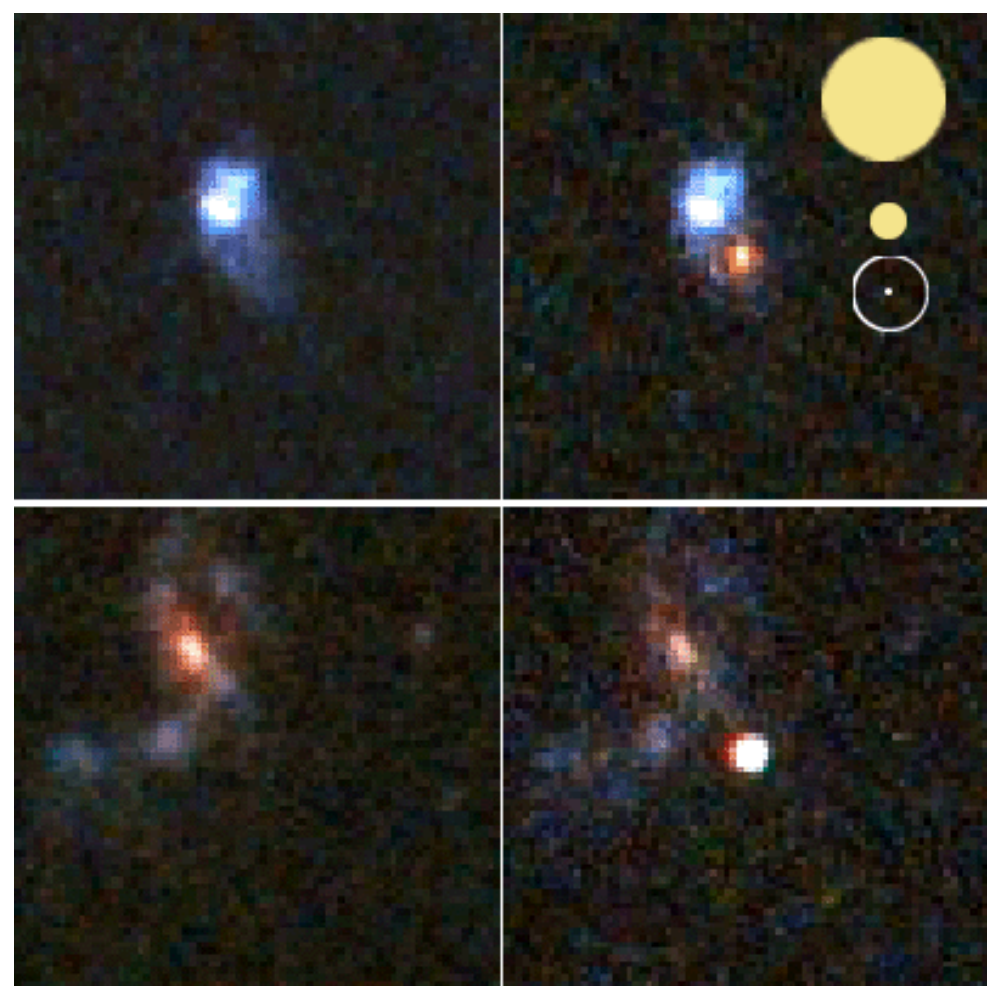

Figure 1. HST ACS images of two $z>1$ supernovae. The PSF of a typical ground-based imaging program, ACS in the $I$-band and a diffraction-limited $H$-band imager on the $24 \mathrm{~m}$ telescope are indicated by the circles in the upper right panel. The ELT PSF has been circled for clarity. ELTs working at the diffraction-limit will overcome confusion with the host galaxy and, when coupled with IFU spectrometers, allow effective spectroscopy of both the SNe and their host galaxies.

telescopes rather than ELTs, although there may be a role for ELTs in studies of acoustic oscillations at $z>4$ if the lower redshift experiments suggest that $\mathrm{w} \neq-1$ and thus motivate probes of the evolution of the dark energy at early times.

\subsection{ELTs and type Ia supernovae}

Type Ia SNe provided the first evidence for an accelerating expansion and they continue to provide one of the pillars of the dark energy paradigm. While the primary sensitivity to $\Lambda$ (if this is the correct explanation) lies at moderate redshifts, SNe at $z>1$ provide a key constraint on evolution and provide a critical check on systematic errors in the Hubble diagram. Presently the best detections and light-curves of distant Type Ia SNe come from the Hubble Space Telescope, as confusion with the light from the host galaxy is a limiting factor. While image differencing techniques can separate the unresolved SN signal from the host galaxy, they are no substitute to a fully resolved image, as illustrated in Figure 1.

ETLs may be able to make an important contribution in this area. Operating at the diffraction-limit in $J$-band, a 30m class ELT will have an order of magnitude greater angular resolution than HST has in the $I$-band, and will sample the rest-frame $V$-band light curves of SNe at $z \sim 1$.5. Higher redshift SNe can be tracked through the $H$ - and $K$-bands allowing one to construct a Hubble diagram within a fixed rest-frame bandpass (e.g. $V$-band) to $z \sim 3$. The challenge may lie in obtaining precise photometry with 
the AO PSF, but this problem pervades so much of the ELT, and even 8-m class, AO science that it will undoubtedly be the focus of considerable attention. The spectroscopy required for accurate spectral classification, host galaxy properties and spectral evolution should be straightforward with an Integral Field Unit (IFU) equipped AO-fed near-IR spectrometer on an ELT. Presently the best spectral classifications for $z>1.5 \mathrm{SNe}$ are achieved with the slitless prism on board ACS/HST. Ground-based ELTs and the James Webb Space Telescope will provide rest-frame visible spectra of SNe at high redshift and allow direct comparison with the spectra of the $z \sim 0.3-0.5 \mathrm{SNe}$ that are critical to dark energy studies long after HST has been retired.

\subsection{Other applications of ELTs to cosmology}

There are a number of other interesting applications of ELTs to cosmology, many of which are described elsewhere in this volume. Programs to measure, or constrain, the evolution of the fine structure constant and other physical constants will be greatly enhanced by the large light grasp of an ELT. Similarly, direct measurements of the cosmic expansion via evolution in the redshifts of $\operatorname{Ly} \alpha$ forest lines require very large collecting areas. These experiments not only require an extremely large telescope, they make very challenging demands on the instruments and their stability.

\section{Galaxy evolution studies with ELTs}

The formation and evolution of galaxies is intimately tied to the cosmic geometry, the nature of dark matter, and the spectrum of seed fluctuations. The diffuse backgrounds and globally-average quantities provide an interface between studies of galaxy evolution and cosmology. They set the ultimate energy constraints, while volume-averaged measures of star formation, accretion onto compact objects, heavy element production and mass assembly provide rate metrics for the growth of galaxies and black holes. Our understanding of the diffuse backgrounds ranges from quite good in the X-ray, where $>90 \%$ of the background is accounted in resolved sources (e.g. Giaconni et al. 2001), to poor in the IR where the luminosity density itself remains rather uncertain. The evolving global quantities provide one approach to understanding which epochs contribute most to any particular part of the unresolved background and a check for missing sources or sinks. A complete understanding of galaxy formation and evolution on cosmologically representative volumes would correctly account for the evolution of the star formation rate, the growth of stellar mass and the evolving chemical content of the Universe.

\subsection{Global measures of galaxy assembly}

The best-known of the global census measures is the star formation rate density (e.g. Madau et al. 1996). While there is close to a consensus regarding the basic picture, the star formation density at $z>5$ is poorly constrained. The complement of the star formation rate history is the evolving stellar mass density (e.g. Dickinson et al. 2003; Glazebrook et al. 2004). The run of stellar mass with redshift shown in Figure 2 reveals that there is an abundance of massive galaxies at $z>1$ and that there are $M>M^{\star}$ galaxies in place at $z \sim 2$. Many of these massive galaxies have spectra dominated by stars with ages of $1-3 \mathrm{Gyr}$ and older, pointing to an early formation epoch for their constituent stellar populations and perhaps for the galaxies themselves (McCarthy et al. 2004; Cimatti et al. 2004). Figure 2 also illustrates the limits to our knowledge of the evolution of $M<M^{\star}$ galaxies at redshifts beyond one. Ground-based ELTs with reasonably large fields of view will be well placed to explore the evolution of a representative range of galaxy masses over the critical redshift range from $1<z<5$. 


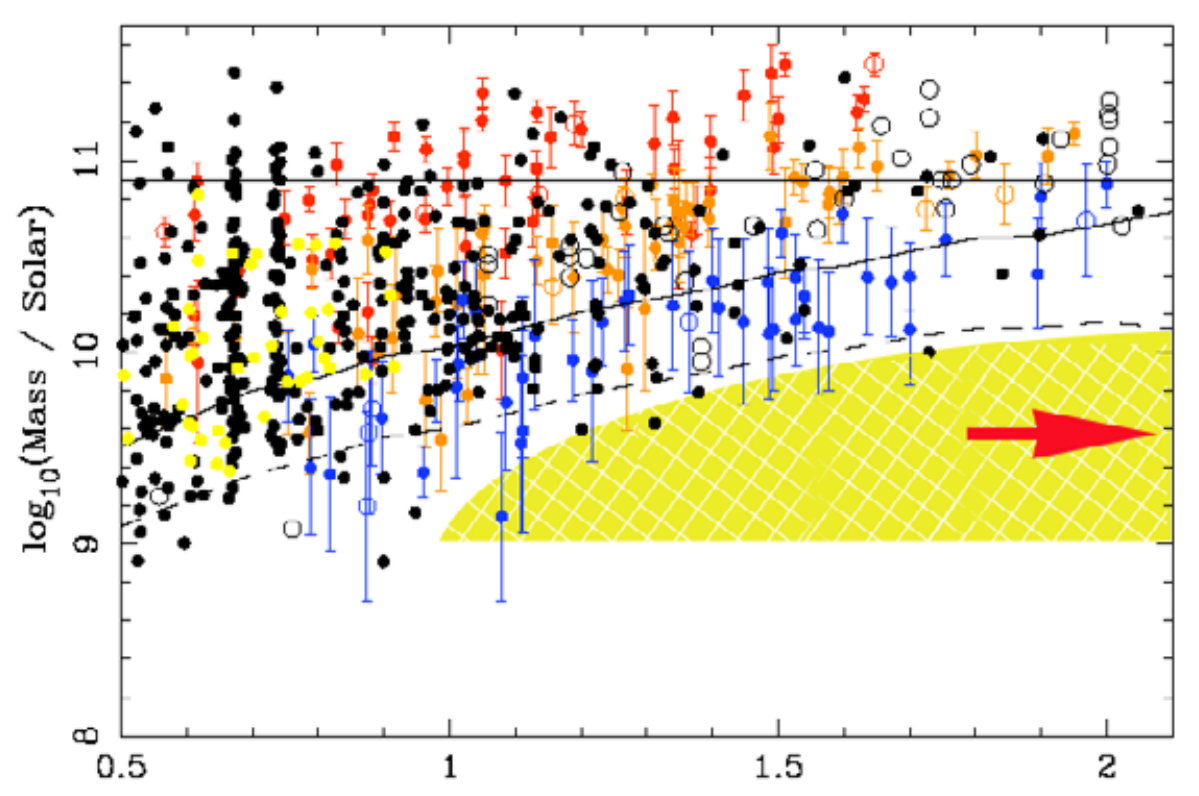

Redshift.

Figure 2. Stellar masses for $K$-band selected galaxies samples in the $0.5<z<2.0$ range from the Gemini Deep Deep and K20 surveys. The present day value of $\mathrm{M}^{*}$ is shown as the solid horizontal line. $K$-band detection limits of 20 and 20.6 Vega magnitudes are shown. The hatched area shows the region of parameter space that can be opened by ELTs working in the visible and near-IR. By accessing the sub-M* $\mathrm{M}^{*}$ regime at $1.5<z<3$, ELTs will be able to directly test hierarchical models for the assembly of massive early-type galaxies. Adapted from Glazebrook et al. (2004).

The hatched region of Figure 2 shows the new area that a $30-m$ class ELT can open up. By probing the $M<M^{\star}$ regime at high redshifts we will be able to critically test hierarchical models of the formation of massive galaxies. In this picture the progenitor to the massive galaxies at $z \sim 0.5$ lie in the hatched, and as yet unexplored, region of the $\mathrm{M}_{\text {star }}-z$ diagram.

Our census of stellar masses at $z>1$ is not only incomplete, it is also likely biased against objects with high $M / L$ values. The most secure determinations of stellar mass content come from spectral synthesis fits to multi-color photometry of well defined samples of galaxies with spectroscopic redshifts. High $M / L$ objects tend to fall below the spectroscopic limits of most surveys, even if they are bright in the near-IR. This is most apparent in surveys of the red galaxies at $z>2$ (e.g. Labbe et al. 2005). Secure redshifts are only available for the high-mass star-forming subset of this population. Considering that this population likely accounts for $1 / 3-2 / 3$ of the total stellar mass density at $z \sim 3$, the case for deeper spectroscopy is quite strong. An ELT working in the red end of the visible or in the near-IR can obtain redshifts from photospheric features, thus removing the bias against passively evolving and obscured systems.

Star formation in galaxies not only leads to a build up in stellar mass, it also produces a changing chemical content in stellar, interstellar and intergalactic matter. Our understanding of the chemical content of galaxies at high redshift is in its infancy. There are a number of challenges, both technical and procedural. Accurate abundance determinations at $z>1.5$ will require precision spectroscopy of the nebular lines as they are 


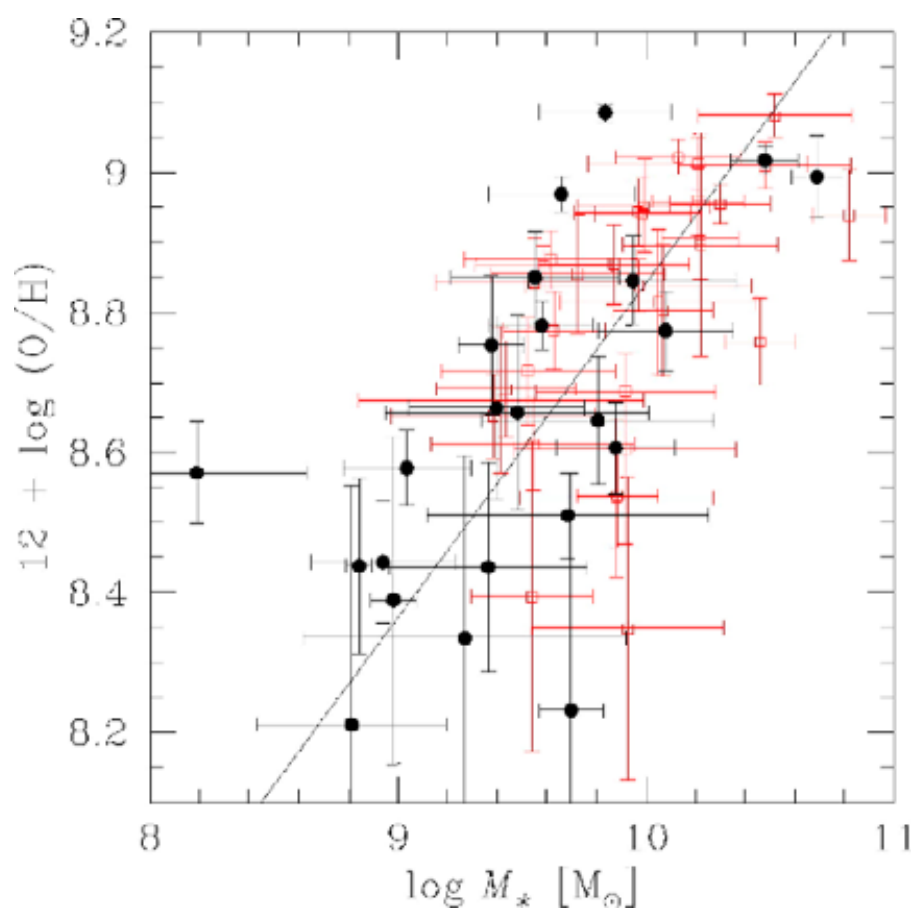

Figure 3. The mass-metallicity relation at $z=0.7$ as derived from the Canada-France redshift survey and the Gemini Deep Deep Survey. The mass-metallicity relation provides a powerful tool for connecting star formation histories, mass growth, and chemical evolution studies of distant galaxies with their present day descendants. ELTs using visible and near-IR spectrometers will sample a broad range of masses and abundances in the critical era of galaxy building. From Savaglio et al. (2005).

redshifted through the near-IR atmospheric windows. Fortunately there are favorable ranges of redshifts for which most of the critical lines are in clean windows. Presently this work is limited to single-object studies of systems with high star formation rates. As IR detectors and spectrometers improve, an ELT with a moderately wide field near-IR spectrometer can carry out an abundance survey over representative volumes and a fair range of galaxy types to allow us to add this powerful diagnostic to the arsenal of tools used in evolution studies. Diffraction-limited IFU imaging spectrometers will be critical here as they will allow detailed examinations of the internal structure of galaxies that can be related to the unresolved quantities derived for large samples. A significant operational challenge in using abundance studies as part of a complete understanding of galaxy evolution is the age-old question of progenitors and descendents. The luminosity-metallicity and mass-metallicity relations provide a means of connecting high redshift progenitors with their present day descendants. The mass-metallicity relation is the more powerful of the two as mass is a more smoothly varying (and likely monotonic) function of time than luminosity. Figure 3 shows a recent determination of the mass-metallicity relation at $z=0.7$ from Savaglio et al. (2005). The existence of a clear mass-metallicity relation at this redshift already provides a probe of the validity of closed box models. Extension to the prime galaxy building epoch will provide a powerful tool for linking the star formation and stellar mass studies. There are indications of relatively high abundances in Lyman break galaxies at $z \sim 2-3$ (Shapley et al. 2004). We know much less about the abundances in the massive red galaxies in the same redshift range. While there are some 


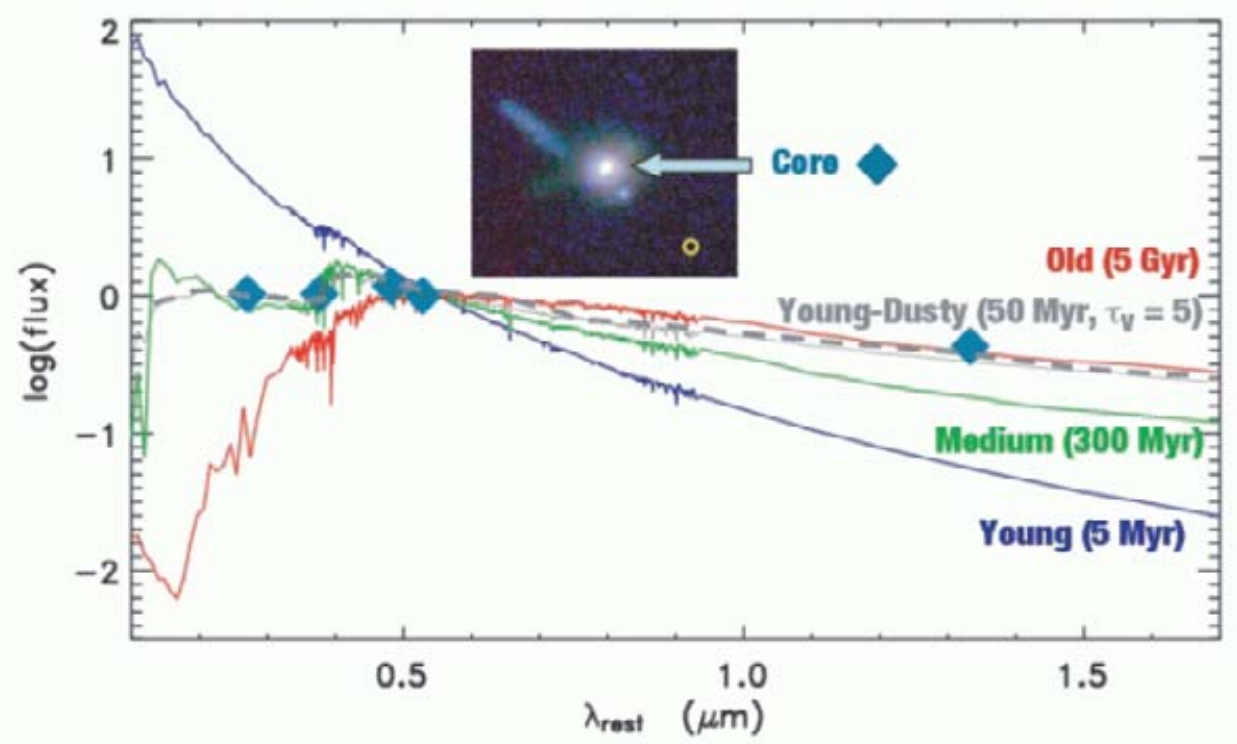

Figure 4. Spectral and spatial decomposition of an intermediate redshift galaxy based on HST visible and Keck near-IR AO images. The figure illustrates the present state of what is rapidly becoming a powerful technique for probing the internal structure and histories of distant galaxies. An AO-fed IFU will allow one to combine dynamical, structural and chemical studies of galaxies over a wide range of redshifts and Hubble types. An ELT is required for the technique to yield its full potential over a broad range of galaxy luminosities and redshifts. From Melbourne et al. (2005).

indications of moderately high abundances in the massive old galaxies at $z \sim 1.5$, the connection between these objects and the $z \sim 3$ population remains unclear.

\subsection{Dissecting the internal structure of galaxies}

The accountant's view of galaxy formation - tracking the flow of baryons from gas to stars and back, while an important test of any model, does not provide a complete picture of galaxy formation. The rich structure of spiral disks, bulges, and halos derives from physics on scales smaller than the cosmological volumes required for representative surveys. Presently we are only able to examine the internal structures of the nearest galaxies, and even in these cases we are often frustrated in our desire to resolve constituent stellar populations. ELTs operating at the diffraction-limit will not only provide unprecedented opportunities for studies of stellar populations in nearby galaxies, they will allow detailed observations of the internal structure of galaxies at high redshift. IFU based determinations of internal dynamics on the $\sim 100 \mathrm{pc}$ scale will allow us to determine total masses, examine disk evolution, and probe recent merger events. The same data will enable studies of star formation modes, duty cycles, the IMF in a range of environments, and the chemical evolution of different components of galaxies across the full range of Hubble types. One of the challenges that we face today is understanding in detail how we arrived at the bimodal galaxy distribution (e.g. Kauffman et al. 2004) wherein the massive galaxies are largely red, inactive and dynamically hot, while the lower mass systems are active and rotationally supported.

Adaptive optics systems on the current generation of 8-10m telescopes are just beginning to explore these techniques. Figure 4 shows an example from the Keck AO system. 

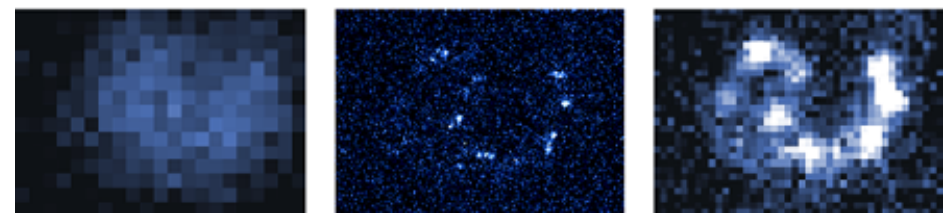

Figure 5. Simulated emission-line images of an interacting galaxy at $z=3.3$ as seen with a $20 \mathrm{~m}$ telescope in 8-hours of integration. The images shown correspond to $0.5^{\prime \prime}$ seeing, diffraction-limited AO, and ground-layer AO with a $0.15^{\prime \prime}$ FWHM PSF. From Barton et al.

In this example Melbourne et al. (2005) perform a spatial and spectral decomposition of an apparently merging system into old (5Gyr) and young $\left(<5 \times 10^{7} \mathrm{yr}\right)$ and dusty components using $K$-band AO images with angular resolutions similar to those of the HST visible images. One will shortly be able to bring spectroscopic capabilities at the same spatial resolution to bear on this problem. This will allow coupling of dynamical and star formation histories.

Spectroscopic studies at the diffraction-limit with 8-m class telescopes will fairly quickly exhaust the range of objects with sufficiently high surface brightness levels. ELTs will provide greater sensitivity, but for some applications one may need to sample the focal plane with pixels larger than the diffraction-limited PSF to achieve sufficient photon rates. This raises the issue of which $\mathrm{AO}$ modes are best suited to the problem of understanding the internal dynamics and structure of galaxies. In Figure 5, I show three simulated images of interacting galaxies at $z=3.3$. The panels show the expected signal-to-noise and resolution for an 8-hour integration on a $20 \mathrm{~m}$ telescope with $0.5^{\prime \prime}$ FWHM natural seeing (left), a diffraction-limited AO PSF (center) and Ground Layer Adaptive Optics (GLAO, $0.15^{\prime \prime} \mathrm{FWHM}$ ). The natural seeing image is clearly inadequate. The diffraction-limited image, while well matched to the size of individual giant HII regions, has little sensitivity outside the largest star forming regions. As one moves to larger apertures, the $\mathrm{S} / \mathrm{N}$ ratio in these regions improves and if one can construct a $50 \mathrm{~m}$ aperture the images get quite good indeed. The right panel in Figure 5 shows a simulated image using a PSF that one might hope to achieve with ground-layer adaptive optics. The GLAO image has quite a bit less resolution than the full AO image; several of the HII regions are blended. The sensitivity to the full structure in the GLAO image, however, is far better than that in the full AO image. It is unclear at this time as to which is superior - the full AO image with its high resolution but low sensitivity to resolved structures, or the GLAO image with its lower resolution but better surface brightness limits. Folding in the larger field of view for ground-layer correction, may well make GLAO the best choice for a wide range of applications.

\subsection{Early galaxies, first light and reionization}

It is fairly clear now that there was a major epoch of galaxy building spanning the redshift range from $z \sim 5$ to $z \sim 1$. It is equally clear that there was substantial activity at higher redshifts, both in terms of star formation and black hole formation and growth. Exploring this early epoch is a key goal for the Webb Telescope and all of the ELT projects. At this time our empirical knowledge of events between recombination at $z=$ 1200 and the end of reionization at $z \sim 6.5$ is limited to a handful bright quasars and faint galaxies and the inferences that can be drawn from their absorption and emission spectra. Starting once again with the global quantities, we can see from Figure 6 that the energy density at $z \sim 6$ and beyond is highly uncertain. Various determinations of the luminosity function have significant differences and the corrections for extinction are 


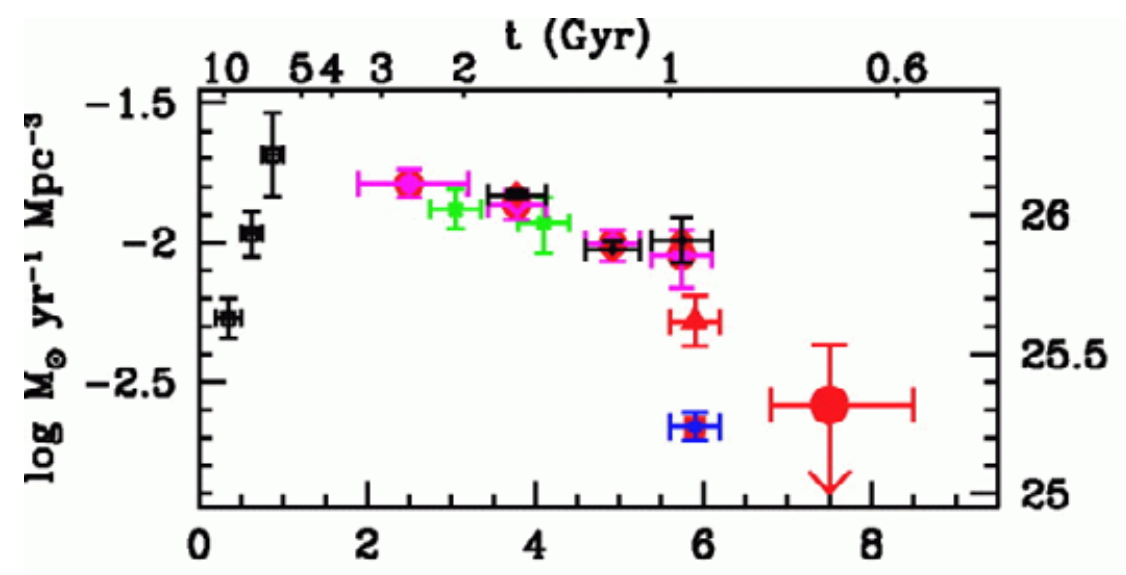

Figure 6. The evolving star formation rate from $z \sim 7$ to the present. The star formation rate at high redshifts remains quite uncertain. While this era contributes little to the integrated total, it is a key probe of structure formation and reionization. Thus there is great interest in exploring this epoch with ELTs and the JWST. From Bouwens et al. (2004).

also large and uncertain. Most of what we know about the faint-end slope is derived from photometric selection of samples that are beyond the reach of spectroscopy with current instruments. An ELT working in the red end of the visible spectrum can provide sensitivity gains of 2-3 magnitudes depending on aperture, reaching well into the sub-L* region of the luminosity function. The limit of practical exposure times has, or soon will be, reached with the current generation of instruments. Experiments with per-source exposure times on the order of 100 hours are either complete or are underway. Clearly, improved sensitivity will require larger collecting areas and, as this work is targeted primarily at the red end of the visible spectrum, there is little prospect of gains from adaptive optics. An ELT with a reasonably wide-field can provide additional information via studies of the evolution of clustering, as the Subaru deep survey has done at $3<z<5$ with SuprimeCam (Ouchi et al. 2005).

There are both theoretical and empirical reasons to believe that there was significant star formation at very early epochs, at redshifts of $\sim 10$ or so. These include numerical hydrodynamic simulations, possible detection of significant optical depth to Thompson scattering at high redshift (Bennett et al. 2003), and the detection of large stellar masses and intermediate ages in $z \sim 6$ galaxies from mid-IR photometry with the Spitzer Space Telescope (e.g. Eyles et al. 2005; Yan et al. 2005; Mobasher et al. 2005). It is thus of great interest, both for ELTs and for the JWST, to understand how one might detect the signature of such early star formation. E. Barton and the US Giant Segmented Mirror Telescope science working group have used numerical simulations by Dave et al. to compute the Ly $\alpha$ flux density for $z \sim 10$ galaxies and the expected $\mathrm{S} / \mathrm{N}$ ratio for a range of ELT apertures. The detectability of the star forming regions at the intersections of cooling filaments, and the diffuse emission from the filaments themselves, depends strongly on the escape fraction for Ly $\alpha$ photons. For this reason Barton et al. (2004) argue that HeII 1640 may be a better signature, as it is not a resonant line. The equivalent width of HeII 1640 is very sensitive to the upper end of the IMF. If the IMF is very top heavy there are reasonable prospects for detecting the galaxies predicted by these models, either through spectroscopy or narrow-band imaging in the near-IR. If the IMF is of Salpeter type, the prospects for detecting the HeII emission, even with a 50m aperture, appear rather discouraging. 


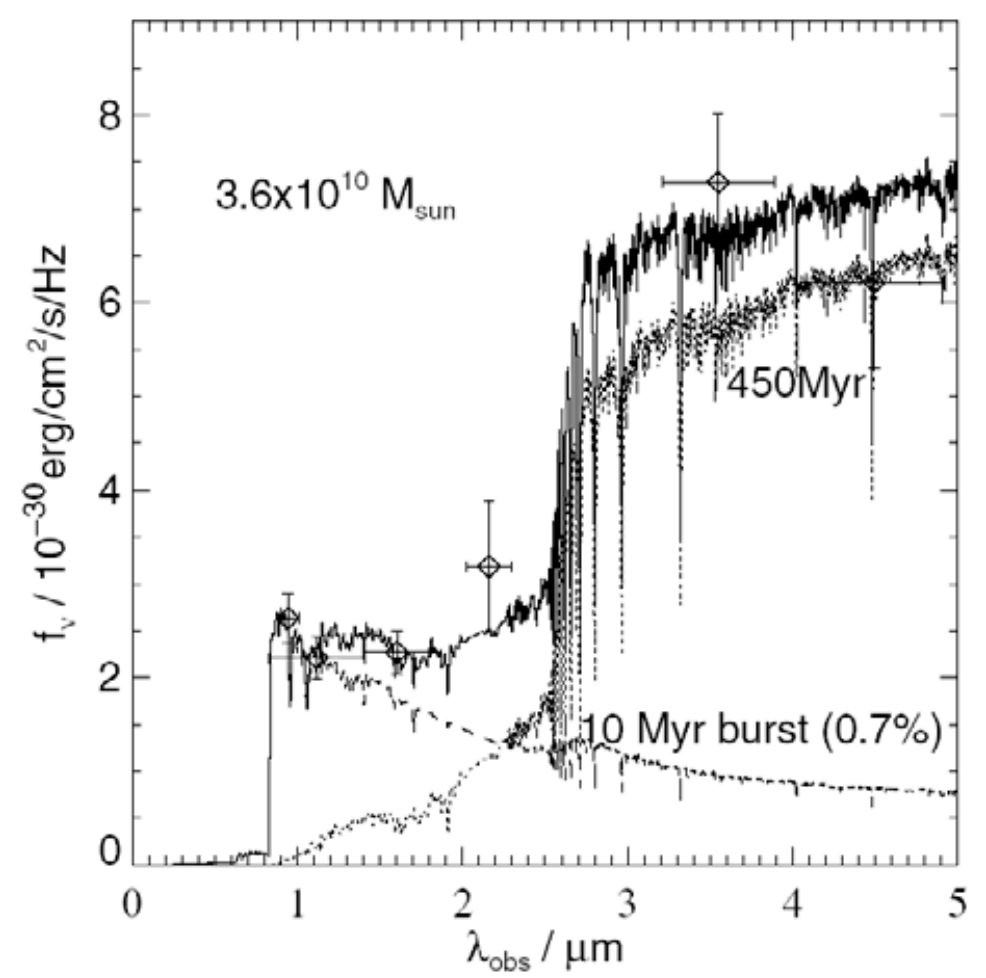

Figure 7. The spectral energy distribution and model fits for a $z=5.8$ Ly $\alpha$ emitting galaxy. The best fitting model is a composite of a $450 \mathrm{Myr}$ old galaxy with a mass of $3.6 \times 10^{8} \mathrm{M}_{\odot}$ and a minority star forming component. The high mass and intermediate age of this object point towards an early epoch of intense star formation. From Eyles et al. (2005).

While the simulations are somewhat sobering regarding the prospects for detections of $z \sim 10$ galaxies, one might take heart from the empirical properties of the known galaxies at $z \sim 6$. Figure 7 shows the best fitting spectral synthesis to one of the $z \sim$ 6 galaxies detected with Spitzer by Eyles et al. The large mass $\left(3.6 \times 10^{10} \mathrm{M}_{\odot}\right)$ and intermediate age $(450 \mathrm{Myr})$ imply a formation redshift of $\sim 10$. Constant SFR models yield star formation rates of $\sim 30 \mathrm{M}_{\odot}$ per year, while more plausible burst models yield rates an order of magnitude larger. The objects detected by Eyles et al, Yan et al. (2005), and others point to an early epoch in which galaxies with mass of a few tenths of $\mathrm{M}^{*}$ were formed. Mobasher et al. (2005) and Wicklind et al. (these proceedings) have identified a population of candidate $z \sim 6$ objects with masses nearly an order of magnitude larger. Presently the redshifts for these objects are inferred from their photometry and so there is greater uncertainty regarding these objects than the objects with secure Ly $\alpha$ redshifts. If the redshifts and ages of these objects are confirmed there is good reason to be quite optimistic about the prospect of directly detecting star forming galaxies in the first Gyr of cosmic time.

\section{Summary and outlook for the future}

We are currently in a golden age for cosmology and faint galaxy studies. There are very real prospects for securing the cosmological parameters with great precision and yet 
we are faced with the mystery of the accelerating expansion. HST and the $8 \mathrm{~m}$ telescopes have given us access to the epoch of galaxy assembly, ELTs and the Webb telescope promise to open a window on the era of first light and reionization. There are a number of ELTs project in the development phase at the moment and these instruments each have a role to play in studies of the distant Universe. It is far too early to know which will succeed and there are abundant examples from the $8 \mathrm{~m}$ era to make the case that the astronomical community would be best served by following more than one approach to the next generation of faint object telescopes.

\section{Acknowledgements}

I thank E. Barton of the GSMT Science Working Group and the members of the GMT Science Working Group (X. Fan, K. Gebhardt, G. Hill, J. Huchra, S. Kenyon, M. Meyer $\&$ A. Weinberger) for allowing me to present some of their work relating to ELT science.

\section{References}

Barton, E., Dave, R., Smith, J., Papovich, C., Herquist, L. \& Springel, V. 2004, ApJ 604, 1

Bennett, C., Halpern, M., Hinshaw, G., Jarosik, N. \& Kogut, A. 2003, ApJS 148, 1

Blake, C. \& Glazebrook, K. 2003, ApJ 594, 665.

Bouwens, R., Thompson, R., Illingwoth, G., Franx, M., van Dokkum, P. et al. 2004, ApJ 616, 79

Cimatti, A. et al. 2004, Nature 430, 184

Dickinson, M., Papovich, C., Ferguson, H. \& Budavari, T. 2003, ApJ 587, 25.

Eyles, S., Bunker, A., Stanway, E., Lacy, M., Ellis, R. \& Doherty, M. 2005, MNRAS 364, 443.

Glazebrook, K., Abraham, R., McCarthy, P., Savaglio, S., Chen, H.-W. et al. 2004, Nature 430, 181.

Giaconni, R., Rosati, P., Tozzi, P. Nonino, M. \& Hasinger, G. 2001, ApJ 551, 624

Labbe, I., Huang, J., Franx, M., Rudnick, G. et al. 2005, ApJ 624, 81

Madau, P., Ferguson, H., Dickinson, M., Giavalisco, M., Steidel, C. \& Fruchter, A. 1996, MNRAS 283,1388

McCarthy, P., Le Bourgne, D., Crampton, D., Chen, H.-W., Abraham, R. et al. 2004, ApJ 614, 9

Melbourne, J., Koo, D. \& Le Floc'h, E. 2005, ApJ 632, 65

Mobasher, B., Dickinson, M., Ferguson, H., Giavalisco, M., Wiklind, T. et al. 2005, ApJ 635, 832

Ouchi, M., Hamana, T., Shimasaku, K., Yamada, T. et al. 2005, ApJ 635, 117

Savaglio, S., Glazebrook, K., Le Borgne, D., Juneau, S. et al. 2005, ApJ 635, 260

Shapley, A., Erb, D., Pettini, M., Steidel, C. \& Adelberger, K. 2004, ApJ 612, 108

Wicklind, T. 2006, these proceedings

Yan H.J., Dickinson, M., Stern, D., Eisenhardt, P. et al. 2005, ApJ 634, 109.

\section{Discussion}

ZINNECKER: I would like to come back to the issue of AGNs, in particular their possible origin in very high-z massive galaxies. There is a big question whether AGN/SMBHs came first or whether the galaxies came first, or whether they came formed together. I wonder how we can decide this question with ELT observations?

MCCARThy: This is an interesting issue, I refer you to the talk by Laura Ferrarese that will occur on Thursday.

FRITZE: I like to make a stronger point than you did in favour of multi-band imaging. For telescopes of any size the vast majority of detectable galaxies will be too faint for 
spectroscopy. Multi-band photometry in combination will Evolutionary Synthesis modelling does not only give photometric redshifts to $5-10 \%$ accuracy but at the same time we have information about the galaxies: ages, SFHs, metallicities, extinction, masses, etc... for all galaxies in deep fields, if only enough wavelength bands are covered.

MCCARTHY: I agree that photometric redshifts are a powerful tool, but there is a limit to how much information one can extract from them. Mass and age determinations are not very precise when the redshift needs to be fitted simultaneously.

FEAST: How good do you think the models are?

MCCARThy: Generally I believe that they are good, but they are limited by the spectral libraries. We need better UV and IR stellar spectral libraries.

CRAmpton: Lensing wasn't mentioned as a tool for probing the very high redshift Universe. Why not, because I am personally afraid that such objects may be too faint for even ELTs?

MCCARTHY: I've long been concerned that the small areas probed by strong lensing limits our ability to draw meaningful conclusions from the results, as spectacular as they are.

Hook: The opticon ELT Science Workimg Group has also been thinking along similar lines to the suggestion you presented, i.e. that GLAO might be the most suitable type of AO for high-z galaxies in terms of matching the PSF to the size of the source (particularly since we require wide fields in order to study large samples). But there is some concern that GLAO may deliver PSFs with only a very small amount of energy in the diffraction limited core, and still leave a large uncorrected halo. Was the PSF used in the high-z galaxy observation simulations by E. Barton a 'realistic' GLAO PSF?

MCCARThy: She assumed that GLAO produced a seeing-like PSF that was a factor of 3 smaller. I agree that it is critical that we understand what GLAO can produce in terms of a PSF. 